\title{
Chronic encephalomyelitis presenting as chronic progressive myelopathy
}

\author{
TORU YAMAMOTO,${ }^{*}$ JOSEFINA F LLENA,${ }^{*}$ EUN SOOK KIM,$\dagger$ ASAO HIRANO $*$ \\ From Bluestone Laboratory of the Division of Neuropathology, ${ }^{*}$ Montefiore Medical Center, Albert Einstein \\ College of Medicine, and the Department of Pathology, $\dagger$ North Central Bronx Hospital, Bronx, New York, \\ USA
}

SUMMARY Chronic progressive myelopathy is a clinical entity with few neuropathological studies. The most frequent diagnosis in necropsied cases is multiple sclerosis. A case of chronic progressive myelopathy is described with a 16-year course in a woman who was 28 years old at the onset of the disease. At necropsy, there was chronic inflammation in the central nervous system with predominant involvement of the spinal cord with diffuse myelin loss and axonal degeneration.

Gradual progression of spastic paraparesis over many years, without additional evidence of pathology in the nervous system, is a perplexing problem for the neurologist. ${ }^{-3}$ Paty et al l $^{1}$ reported a clinical study of patients diagnosed as chronic progressive myelopathy who had gradual progression of spastic gait, hyperactive reflexes, and variable degree of sensory loss. Using the parameters of oligoclonal bands in cerebrospinal fluid (CSF), visual evoked responses, and blink reflexes, they disclosed one or more abnormalities in $85 \%$ of the cases and interpreted them as probably multiple sclerosis.

In one necropsy series, 12 of 35 patients who presented with spastic paraplegia of uncertain aetiology were shown to have multiple sclerosis. ${ }^{2} \mathrm{~A}$ variety of diagnoses were made in the other cases, such as intraspinal tumour, syringomyelia, and aneurysm of the intervertebral spinal artery. In a case of typical chronic progressive myelopathy, we describe yet another disease entity, an inflammatory process in the entire neuraxis, with predominant and severe involvement of the spinal cord.

\section{Case report}

A hospital ward attendant was well until 1967 , at the age of 28 , when she had acute onset of low back pain radiating down the posterior aspect of both legs after helping to lift a heavy patient. In spite of several weeks' rest the pain did

Address for reprint requests: Toru Yamamoto, MD, Bluestone Laboratory of the Division of Neuropathology, Montefiore Medical Center, 111 East 210th Street, Bronx, NY 10467, USA

Received 6 January 1984 and in revised form 13 April 1984. Accepted 18 April 1984 not improve and she was admitted to a hospital. Examination revealed $3+$ knee jerk and $2+$ ankle jerk bilaterally, and pain on straight leg raising on the left. The rest of the examination including muscle power and sensation was normal. Laboratory findings was also negative. A diagnosis of lumbar disc herniation was made and she returned to work. The pain did not cease during 1968 and 1969. Meanwhile, limping and some weakness of the right leg developed insidiously. She was re-admitted in 1969 for further study. Neurological examination disclosed weakness of the right leg, bilateral ankle clonus, and bilateral Babinski signs. Myelogram, pneumoencephalogram, brain scintiscan, and EMG were normal. Laboratory study was again within normal limits. The lumbar puncture yielded CSF that contained $36 \mathrm{mg} / \mathrm{dl}$ protein and 6 white blood cells per $\mathrm{mm}^{3}$; the differential of the latter was unavailable. After discharge without a definite diagnosis, weakness and stiffness in both lower extremities continued to worsen.

The patient was admitted to Montefiore Medical Center in 1972 for further evaluation. At that time she described muscle spasm with twitches in both legs, "pins and needles" sensation in both feet, numbness and coldness of both legs and feet, and some urinary urgency. On examination she was alert, oriented, and co-operative. Cranial nerves were intact. Optic fundi were normal. Motor and sensory examination of the arms was normal but there was spasticity and moderate weakness in the legs, with wasting of the small muscles of the feet. The tendon reflexes were $3+$ in the arms, and $4+$ in the legs with clonus. Both plantar responses were extensor. Sensation was normal in the legs, and there was no ataxia or nystagmus. She needed a cane to walk. A myelogram was normal and routine haematological, immunological and biochemical studies were also normal. The CSF IgG was $33 \mathrm{mg} / \mathrm{dl}, \operatorname{IgA}$ and IgM were negative, but the cell count and total protein were not reported. A clinical diagnosis of probable multiple sclerosis was made. The paraplegia continued to worsen and incontinence of urine developed. She became 


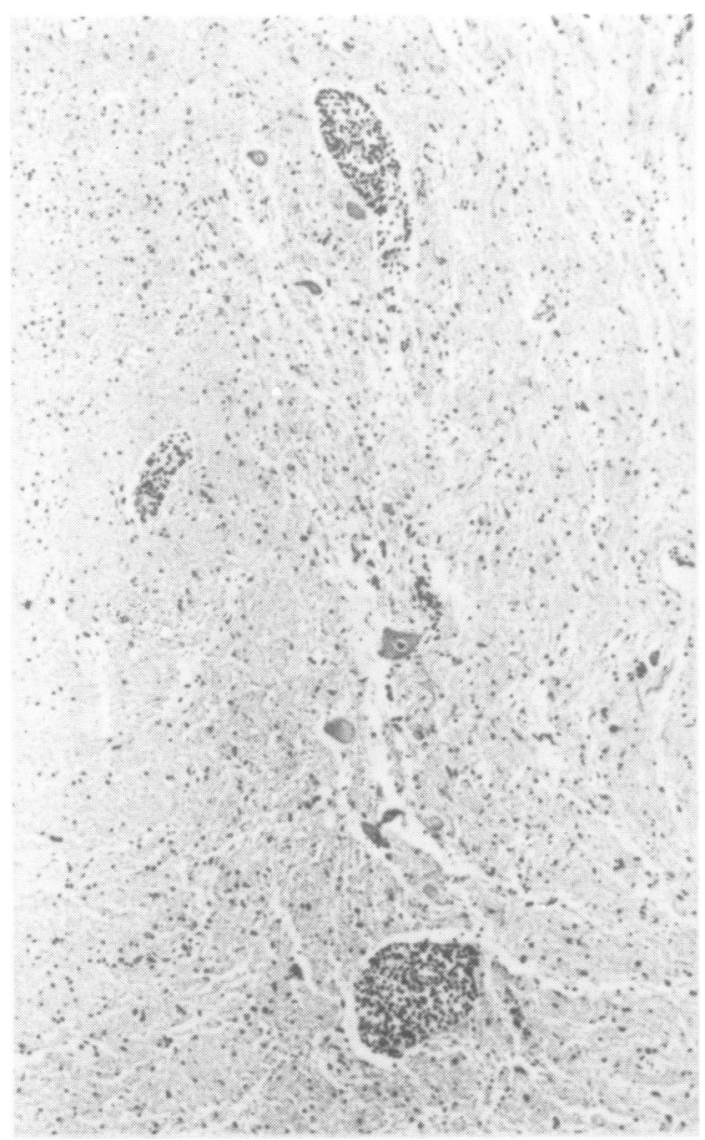

Fig 1 Anterior horn of the thoracic cord, showing perivascular cuffing and scattered parenchymal infiltration of mononuclear cells. Astrocytosis is marked. Anterior horn cells are relatively well preserved. $(H \& E \times 88)$.

bed-ridden in 1977. She died at age 45 years with infected decubitus ulcers.

\section{Pathology}

General necropsy findings included bilateral bronchopneumonia, pulmonary edema, chronic oesophagitis, distension of the small and large intestines with fecal impaction, and generalised visceral congestion. The weight of the unfixed brain was 1050 grams. The brain, brainstem, and their coverings were unremarkable. The spinal cord from $\mathrm{Th}_{1}$ downwards was available for examination. It was atrophic throughout its length. Multiple sections of the spinal cord revealed diffuse, brownish discoloration of the white matter. No discrete multiple sclerosis plaque was identified.

\section{Histopathological findings}

Representative sections of the brain and spinal cord were stained with one or more of the following methods: haematoxylin and eosin ( $\mathrm{H} \& \mathrm{E})$, luxol fast blue-periodic acid Schiff (LFB-PAS), Bodian, Bielschowsky, Holzer, and Sudan IV. There was no plaque characteristic of multiple sclerosis. There was prominent perivascular cuffing and parenchymal clusters of lymphocytes, associated with widespread, ill-demarcated loss of myelin and axon throughout the neuraxis, with strong predilection for the spinal cord. The characteristic features of the lesions consisted of: (a) perivascular cuffing in the white as well as gray matter with mononuclear cells, most of which were small lymphocytes (figs 1,2 ) and rare plasma cells; macrophages were not observed; some clusters of similar cells were scattered in the parenchyma, (b) no apparent relationship between the infiltration of cells and loss of myelin; the latter was widespread and roughly symmetrical (fig 3), (c) axonal degeneration associated with myeline loss, (d) relatively well preserved large neurons; neuronophagia or inclusion bodies were not observed, (e) diffuse astrocytic gliosis in the area of cellular infiltration, ( $f$ ) only mild cellular infiltration in the leptomeninges, $(\mathrm{g})$ well-preserved spinal roots, (h) collagenous thickening of small vessels.

Additional features with regards to topography were as follows: the thoracic cord was atrophic and had most remarkable cellular infiltration. The normal architecture of the cord was obscured. In one section a meningeal vein had a distinct perivascular cuff of lymphocytes. Myelin was almost totally lost, except for the faintly-stained, rather symmetrical areas in the posterior columns (fig 3). At the entry zone of the nerve roots remyelination with peripheral type myelin was observed. The lumbar cord had a similar lesion. In addition, most of its anterior horn cells showed chromatolysis. The normal structure of the medulla was preserved but again loss of myelin was severe, widespread, and symmetrical, especially in the pyramids and amiculi of the inferior olivary complexes. Sudan IV stain revealed no fat deposit. The pontine base had some asymmetry in the distribution of myelin loss. Ependymal lining was normal. Perivascular lymphocytic cuffing was mild in the rest of the brain, and myelin loss was not seen in the cerebrum and optic nerves.

\section{Discussion}

The literature of chronic progressive myelopathy or spastic paraplegia of unknown origin is scanty. In 1955 Marshall $^{2}$ studied clinically 52 cases of such patients diagnosed as "spastic paraplegia of uncertain aetiology". Some ten years after the onset, the cause of the disorder had declared itself in about half the cases $(27 / 52) ; 10$ were multiple sclerosis, seven tumour, and 10 various diseases including an obscure entity, paraplegia with dementia. He also reviewed the necropsy diagnosis in another 35 cases; 12 had multiple sclerosis, 11 tumour, three synringomyelia, two prolapsed disc, two spinal deformity, and five miscellaneous diseases. Hübbe and $\mathrm{Dam}^{3}$ studied 24 patients surviving after the mean observation time of 8.9 years: a diagnosis was reached in 


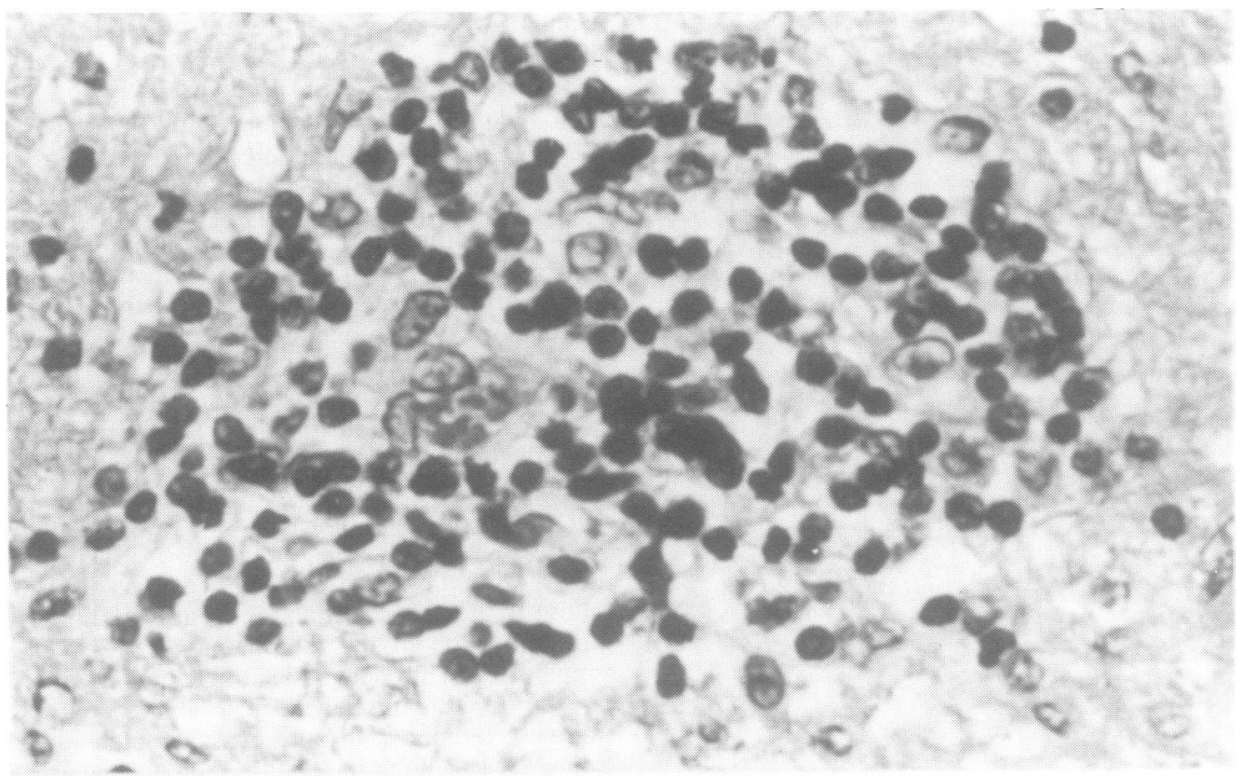

Fig 2 Higher magnification of the perivascular cuffing. Most of the cells are small lymphocytes. (H\&E $\times$ 810).

eight patients only; six were multiple sclerosis, one spinocerebellar degeneration, and one intraspinal tumour. Interestingly, the remaining undiagnosed cases still had an isolated spastic paraplegia that progressed slowly and gradually.

The present case has the typical features of chronic progressive myelopathy. The patient developed a slowly progressive spastic paraplegia without known cause over a long period of 16 years. Even at the time of the last admission, she was oriented, co-operative, and without gross sensory deficit. Definite diagnosis could not be reached clinically and she was regarded as having chronic, progressive multiple sclerosis. There was no abnormality in the laboratory tests, except for high IgG in CSF, though oligoclonal banding and viral studies were not performed.

The differential diagnoses of the pathological picture included chronic encephalomyelitis, primary intramedullary spinal lymphoma, multiple sclerosis,

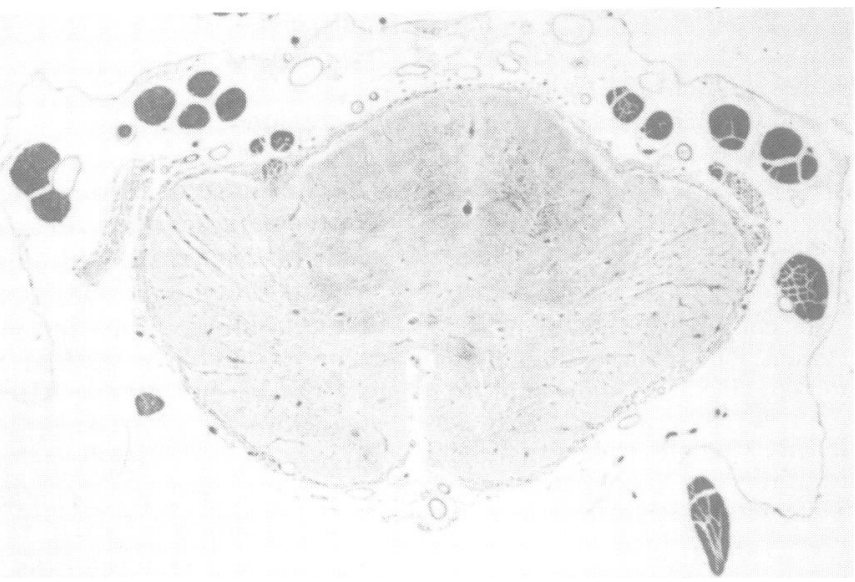

Fig 3 The thoracic cord showing diffuse, widespread myelin loss with some faintly-stained myelin in the posterior columns. In contrast, the spinal roots are well preserved. (LFB-PAS $\times 10)$. 
allergic encephalomyelitis, and other rare diseases.

In multiple sclerosis, axons remain relatively intact in more or less distinct demyelinating plaques, except for some severe or long standing cases in which diffuse long tract degeneration develops. The admixture of plaques, necrosis, and Wallerian degeneration, as seen in some cases of multiple sclerosis, for example Devic type, ${ }^{4}$ produces a picture similar to the present case. In addition, perivascular cuffing is seen not infrequently in multiple sclerosis, either acute or chronic, and sometimes to a remarkable degree. ${ }^{5}$ In our case, however, the lack of discrete demyelinating plaques or relative sparing of axons anywhere in the central nervous system, makes the diagnosis very unlikely. The characteristic perivenular pattern of demyelination observed in allergic encephalomyelitis including rare cases with a chronic course, ${ }^{4}$ was not the picture of our case either.

Primary lymphoma of the cord is a very rare entity that has been reported only in six cases. ${ }^{6}$ The most reliable clinical finding in all cases was segmental widening of the cord on myelography, ${ }^{6}$ which was absent in this case. Among their 25 cases of primary reticulum cell sarcoma of the central nervous system, Schaumburg et al ${ }^{7}$ reported an unusual case of a 46-year-old man who after spontaneous remission of the initial symptoms and signs, lived 8 years with minimal disability before a 7 months terminal illness. At necropsy there was extensive brainstem and thalamic infiltration by tumour. Such a long survival in cases of reticulum cell sarcoma or lymphoma, although rare, warrants its consideration in a chronic case like ours. However, no mass nor neoplastic cells of malignant lymphoma were observed in our case.

Lymphomatoid granulomatosis frequently presents a picture of meningo-encephalitis and may not be an expanding lesion. ${ }^{8}$ It has been defined as a necrotising angiocentric and angiodestructive infiltrative disorder associated with an unpredictable prognosis ranging from long survival to rapid deterioration. In our case, the vessels were unremarkable and systemic involvement was absent.

Among other diseases presenting with perivascular mononuclear cell infiltration are histiocytosis X, familial lympho-histiocytosis of the nervous system, and chronic recurrent demyelinating encephalomyelitis associated with hemophagocytic lympho-histiocytosis. ${ }^{9}$ Those were easily ruled out mainly by the absence of histiocytes.

Adrenoleukodystrophy, a sex-linked recessive disorder, is known to have perivascular cuffing with lymphocytes, plasma cells, and macrophages. ${ }^{10}$ One of the clinical forms is seen in symptomatic heterozygotes, females in their third decade with progresive spastic paraparesis over 20 years." The pathology of such cases has not been reported so far. A negative family history, the absence of a lesion in the adrenal glands, and the lack of the typical neuropathology rule out this diagnosis in our case.

Encephalomyelitis or encephalitis is usually an acute disorder caused by viral, bacterial, fungal, and parasitic infections, or by allergy, but it is rarely chronic as in subacute sclerosing panencephalitis, progressive rubella panencephalitis of human, and visna of sheep. Other types of chronic encephalomyelitis or myelitis have been reported but little is known about their etiology. Under the term progressive encephalomyelitis with rigidity, four cases of progressive encephalomyelitis, with predominance in the spinal cord, have been described.12-15 All were characterised clinically by progressive rigidity and pathologically by perivascular lymphocytic cuffing and parenchymal infiltration, as well as diffuse, more or less symmetrical loss of myelin. The patients were followed 13 months to $3 \frac{1}{2}$ years before death. The remarkable rigidity, muscle spasms, and myoclonus were ascribed to selective destruction of small and medium sized neurons in the ventral horns, most of which were supposed to be spinal internuncial neurons. ${ }^{12}$ This selectivity did not necessarily apply to all cases, however. ${ }^{14}$ is In addition, the pattern of involvement was variable, except for the relatively constant sparing of the lateral corticospinal tracts. Therefore, the basic pathological picture, that is cellular infiltration and widespread loss of myelin and axon, resembles that of our case. The aetiology of progressive encephalomyelitis with rigidity is not known but various kinds of viruses including that of encephalitis lethargica have been suggested..$^{15}$

In this clinico-pathological study, we document a chronic inflammatory process as another aetiology of chronic progressive myelopathy. Additional cases with virological and immunological studies are needed, to elucidate the exact pathogenesis of this entity and to establish whether it forms a spectrum with the reported cases of chronic encephalomyelitis with rigidity.

\section{References}

' Paty DW, Blume WT, Brown WF, et al. Chronic progressive myelopathy: investigation with CSF electrophoresis, evoked potentials, and CT scan. Ann Neurol 1979;6:419-24.

${ }^{2}$ Marshall J. Spastic paraplegia of middle age. A clinicopathological study. Lancet 1955;1:643-6.

${ }^{3}$ Hübbe P, Dam AM. Spastic paraplegia of unknown origin. Acta Neurol Scand 1973;49:536-42.

${ }^{4}$ Oppenheimer DR. Demyelinating diseases. In: Blackwood W, Corsellis JAN, eds. Greenfield's 
Neuropathology. Third edition. Edinburgh: Edward Arnold 1976:470-99.

${ }^{5}$ Guseo A, Jellinger $\mathrm{K}$. The significance of perivascular infiltrations in multiple sclerosis. $J$ Neurol 1975;211:51-60.

'Hautzer NW, Aiyesimoju A, Robitaille Y. "Primary" spinal intramedullary lymphomas: a review. Ann Neurol 1983; 14:62-6.

' Schaumburg HH, Plank CR, Adams RD. The reticulum cell sarcoma-microglioma group of brain tumors. A consideration of their clinical features and therapy. Brain 1972;95:199-212.

${ }^{8}$ Michaud J, Banerjee D, Kaufmann JCE. Lymphomatoid granulomatosis involving the central nervous system: Complication of a renal transplant with terminal monoclonal B-cell proliferation. Acta Neuropathol (Berl) 1983;61:141-7.

${ }^{9}$ Nagashima T, Yamada K. Uono M, et al. Chronic recurrent demyelinating encephalomyelitis associated with hemophagocytic lymphohistiocytosis. Acta Neuropathol (Berl) 1983;59:25-30.

${ }^{10}$ Schaumburg HH, Powers JM, Suzuki K, et al. Adreno-
Leukodystrophy (Sex-linked Schilder disease). Arch Neurol 1974;31:210-3.

"Moser HW, Moser AB, Kawamura N, et al. Adrenoleukodystrophy: Studies of the phenotype, genetics, and biochemistry. John Hopkins Med J 1980; 147:217-24.

${ }^{12}$ Howell DA, Lees AJ, Toghill PJ. Spinal internuncial neurones in progressive encephalomyelitis with rigidity. J Neurol Neurosurg Psychiatry 1979;42:773-85.

${ }^{13}$ Kasperek S, Zebrowski S. Stiff-man syndrome and encephalomyelitis. Report of a case. Arch Neurol 1971;24:22-30.

${ }^{14}$ Lhermitte F, Chain F, Escourolle R, et al. Un nouveau cas de contracture tétaniforme distinct (du stiff man syndrome). Ètude pharmacologique et neuropathologique d'un cas d'encéphalomyélite à prédominance médullaire. Revue Neurol (Paris) 1973;128:3-21.

${ }^{15}$ Whiteley AM, Swash M, Urich H. Progressive encephalomyelitis with rigidity. Its relation to "subacute myoclonic spinal neuronitis" and to the "stiff man syndrome". Brain 1976;99:27-42. 